\title{
Trapped Fermi gases with Rashba spin-orbit coupling in two dimensions
}

\author{
M. Iskin \\ Department of Physics, Koç University, Rumelifeneri Yolu, 34450 Sartyer, Istanbul, Turkey \\ (Received 8 June 2012; revised manuscript received 16 November 2012; published 5 December 2012)
}

\begin{abstract}
Here we consider both balanced and imbalanced Fermi gases throughout the BCS-BEC evolution, and we study the effects of spin-orbit coupling (SOC) on the spontaneously induced countercirculating mass currents and the associated intrinsic angular momentum via the Bogoliubov-de Gennes (BdG) formalism. In particular, we find that even a small SOC destabilizes Fulde-Ferrel-Larkin-Ovchinnikov (FFLO) type spatially modulated superfluid phases and phase-separated states against the polarized superfluid phase. We also show that the continuum of the quasiparticle and quasihole excitation spectrum can be connected by zero, one, or two discrete branches of interface modes, depending on the number of interfaces between a topologically trivial phase and a nontrivial phase.
\end{abstract}

DOI: 10.1103/PhysRevA.86.065601

PACS number(s): 03.75.Ss, 05.30.Fk, 03.75.Hh

Introduction. The coupling between a quantum particle's intrinsic angular momentum (spin) and its center-of-mass (orbital) motion has important consequences in a variety of modern condensed-matter problems, ranging from quantum spin Hall systems to topological insulators and topological superconductors [1,2]. This interaction is referred to as the spin-orbit coupling (SOC), and it arises from coupling of the electron's spin to the local magnetic field that is induced in the electron's reference frame due to the time-varying electric field produced by the charged background. Since both the strength and symmetry of the SOC are mainly determined by the electronic structure of the crystal in condensed-matter systems, it is more desirable to engineer SOC in alternative systems that allow more control over its parameters. Given the recent experimental advances in simulating artificial gauge fields with neutral quantum gases [3-6], it is arguable that the prime candidate for engineering SOCs in a controllable many-body setting seems to be the atomic ones. For instance, this has recently been achieved first with bosonic [3,4] and then fermonic $[5,6]$ atomic gases by coupling the momentum of atoms to their spin via a pair of laser beams. While the symmetry of all of the experimentally engineered SOCs is so far an equal combination of Rashba and Dresselhaus terms, theoretical proposals for creating unequal combinations are also underway.

Since the realization of spin-orbit-coupled Bose-Einstein condensates (BECs) [3], there has been growing theoretical interest in studying spin-orbit-coupled Fermi gases, even prior to their very recent realization $[5,6]$. For population-balanced uniform systems, it has been shown that the BCS-BEC evolution is a crossover, and this evolution can be driven either by increasing the interparticle interaction strength for a fixed SOC or by increasing the SOC for a fixed interaction strength (no matter how small the latter is) [7-12]. On the other hand, for imbalanced systems, the BCS-BEC evolution is not a crossover, and quantum phase transitions are found between thermodynamically stable and topologically distinct gapped and gapless superfluid phases. These phases are distinguished in momentum space by their numbers of zero-energy points, rings, or surfaces (depending on the type of SOC) in their excitation spectrum [13-19].

In direct application to atomic systems, the thermodynamic phase diagrams obtained in these works can be used to extract information about the trapped Fermi gases, at least within the semiclassical local-density approximation [20-23]. While this approximation works better when the number of fermions is large, as the finite-size effects become negligible, a fully quantum-mechanical method, e.g., BdG formalism, is better suited for studying finite-size effects, which is the goal of this work. Here we consider Rashba SOC in two dimensions due to its numerical simplicity and hope that some of our qualitative conclusions hold in three dimensions as well. However, given the recent realization of two-dimensional Fermi gases [24,25], it may also be possible to engineer SOC in reduced dimensions. Our main focus is on the SOC-induced countercirculating mass currents, where we systematically analyze their dependence on the SOC, binding energy, and population imbalance. We note that induced currents have recently been discussed for the lattice model [26], and while the Hamiltonian used and the $\mathrm{BdG}$ formalism developed in this work are completely different, our results are in qualitative agreement with each other when there is an overlap.

$B d G$ formalism. We use the Hamiltonian density (in units of $\left.\hbar=k_{B}=1\right), H(\mathbf{r})=\sum_{\sigma, \sigma^{\prime}} \psi_{\sigma}^{\dagger}(\mathbf{r}) K_{\sigma \sigma^{\prime}}(\mathbf{r}) \psi_{\sigma^{\prime}}(\mathbf{r})+$ $\Delta(\mathbf{r}) \psi_{\uparrow}^{\dagger}(\mathbf{r}) \psi_{\downarrow}^{\dagger}(\mathbf{r})+\Delta^{*}(\mathbf{r}) \psi_{\downarrow}(\mathbf{r}) \psi_{\uparrow}(\mathbf{r})$, where the operators $\psi_{\sigma}^{\dagger}(\mathbf{r})$ and $\psi_{\sigma}(\mathbf{r})$ create and annihilate a pseudospin $\sigma$ fermion at position $\mathbf{r}$, respectively, and $\Delta(\mathbf{r})$ is the mean-field superfluid order parameter. The diagonal operator $K_{\sigma \sigma}(\mathbf{r})=$ $-\nabla^{2} /(2 M)-\mu_{\sigma}+V(r)$ includes both the kinetic energy and the harmonic trapping potential $V(r)=M \omega^{2} r^{2} / 2$, where $M$ is the mass and $\mu_{\sigma}$ is the chemical potential of $\sigma$ fermions, and $\omega$ is the trapping frequency. The off-diagonal operator $K_{\uparrow \downarrow}(\mathbf{r})=K_{\downarrow \uparrow}^{\dagger}(\mathbf{r})=\alpha\left(p_{y}+i p_{x}\right)$ is the Rashba SOC, where $\alpha \geqslant 0$ is its strength and $p_{j}=-i \partial / \partial j$ is the momentum operator. In the polar coordinate system $(r, \theta)$, this term becomes $K_{\uparrow \downarrow}(\mathbf{r})=e^{-i \theta}[\partial / \partial r-i \partial /(r \partial \theta)]$, which makes the Rashba SOC numerically much easier to simulate in two dimensions due to its rotational invariance.

This Hamiltonian can be diagonalized via a generalized Bogoliubov-Valatin transformation, and the resultant $\mathrm{BdG}$ equation is $H(\mathbf{r}) \varphi_{\nu}(\mathbf{r})=\varepsilon_{v} \varphi_{\nu}(\mathbf{r})$, where

$$
H(\mathbf{r})=\left[\begin{array}{lccc}
K_{\uparrow \uparrow}(\mathbf{r}) & K_{\uparrow \downarrow}(\mathbf{r}) & 0 & \Delta(\mathbf{r}) \\
K_{\downarrow \uparrow}(\mathbf{r}) & K_{\downarrow \downarrow}(\mathbf{r}) & -\Delta(\mathbf{r}) & 0 \\
0 & -\Delta^{*}(\mathbf{r}) & -K_{\uparrow \uparrow}^{*}(\mathbf{r}) & -K_{\uparrow \downarrow}^{*}(\mathbf{r}) \\
\Delta^{*}(\mathbf{r}) & 0 & -K_{\downarrow \uparrow}^{*}(\mathbf{r}) & -K_{\downarrow \downarrow}^{*}(\mathbf{r})
\end{array}\right]
$$


is the Hamiltonian matrix given in the $\varphi_{\nu}(\mathbf{r})=$ $\left[u_{\uparrow v}(\mathbf{r}), u_{\downarrow v}(\mathbf{r}), v_{\uparrow v}(\mathbf{r}), v_{\downarrow v}(\mathbf{r})\right]^{\mathrm{T}}$ basis and $\varepsilon_{v} \geqslant 0$ are the energy eigenvalues. The order parameter $\Delta(\mathbf{r})=g\left\langle\psi_{\uparrow}(\mathbf{r}) \psi_{\downarrow}(\mathbf{r})\right\rangle$, where $g \geqslant 0$ is the strength of the interparticle attraction and $\langle\cdots\rangle$ is the thermal average, becomes $\Delta(\mathbf{r})=g \sum_{v}\left[u_{\uparrow v}(\mathbf{r}) v_{\downarrow v}^{*}(\mathbf{r}) f\left(-\varepsilon_{v}\right)+u_{\downarrow v}(\mathbf{r}) v_{\uparrow v}^{*}(\mathbf{r}) f\left(\varepsilon_{v}\right)\right]$. Here, $f(x)=1 /\left(e^{x / T}+1\right)$ is the Fermi function and $T$ is the temperature. We relate $g$ to the energy $\varepsilon_{b} \leqslant 0$ of the two-body bound state between an $\uparrow$ and a $\downarrow$ fermion in vacuum via $1 / g=\sum_{\mathbf{k}} 1 /\left(2 \varepsilon_{\mathbf{k}}-\varepsilon_{b}\right)$, where $\varepsilon_{\mathbf{k}}=k^{2} /(2 M)$ is the kinetic energy. This leads to $g=4 \pi /\left[M \ln \left(1+2 \varepsilon_{c} /\left|\varepsilon_{b}\right|\right)\right]$, where $\varepsilon_{c}$ is the energy cutoff used in the $\mathbf{k}$-space integration $\left(\varepsilon_{c}\right.$ is specified below). $\Delta(\mathbf{r})$ needs to be solved self-consistently with the number equations $N_{\sigma}=\int d \mathbf{r} n_{\sigma}(\mathbf{r})$, where $n_{\sigma}(\mathbf{r})=$ $\left\langle\psi_{\sigma}^{\dagger}(\mathbf{r}) \psi_{\sigma}(\mathbf{r})\right\rangle=\sum_{\nu}\left[\left|u_{\sigma v}(\mathbf{r})\right|^{2} f\left(\varepsilon_{v}\right)+\left|v_{\sigma v}(\mathbf{r})\right|^{2} f\left(-\varepsilon_{v}\right)\right]$ is the local density of $\sigma$ fermions. Thus, the order parameter and number equations form a closed set, determining $\Delta(\mathbf{r})$ and $\mu_{\sigma}$ for any given $\varepsilon_{b}, \alpha$, and $T$.

We emphasize that these mean-field equations can be used to investigate the low-temperature properties of the system for all values of $\varepsilon_{b}$ and $\alpha$, but they provide only a qualitative description of the system outside of the weak-coupling regime, i.e., in the BCS-BEC evolution. Once the quasiparticle energies and the corresponding wave functions are obtained, it is a straightforward task to calculate other observables of interest. For instance, the quantum-mechanical probability-current operator for $\sigma$ fermions can be identified from the continuity equation. While the presence of a SOC leads to additional terms in the total particle-current operator, these terms do not contribute to the current since $\left\langle\psi_{\uparrow}^{\dagger}(\mathbf{r}) \psi_{\downarrow}(\mathbf{r})\right\rangle=0$. Therefore, the local current density $\mathbf{J}_{\sigma}(\mathbf{r})=[1 /(2 M i)]\left\langle\psi_{\sigma}^{\dagger}(\mathbf{r}) \nabla \psi_{\sigma}(\mathbf{r})\right.$ - H.c.) circulating around the center of the trapping potential becomes $\mathbf{J}_{\sigma}(\mathbf{r})=[1 /(2 M i)] \sum_{\nu}\left[u_{\sigma \nu}^{*}(\mathbf{r}) \nabla u_{\sigma \nu}(\mathbf{r}) f\left(\varepsilon_{v}\right)+\right.$ $v_{\sigma \nu}^{*}(\mathbf{r}) \nabla v_{\sigma \nu}(\mathbf{r}) f\left(-\varepsilon_{v}\right)-$ H.c.], where H.c. is the Hermitian conjugate. We note that the intrinsic angular momentum associated with this spontaneous flow is along the $\widehat{\mathbf{z}}$ direction, and its density is given by $\ell_{\sigma}(r)=M r J_{\sigma}(r)$.

Numerical results. In our calculations, we set a large energy cutoff $\varepsilon_{c} \gg \varepsilon_{F}$ and solve the self-consistency equations for different $P=\left(N_{\uparrow}-N_{\downarrow}\right) / N$ values. Here, $\varepsilon_{F}=k_{F}^{2} /(2 M)=$ $M \omega^{2} r_{F}^{2} / 2$ is a characteristic Fermi-energy scale, where $r_{F}$ is the Thomas-Fermi radius and $k_{F}$ is the Fermi momentum corresponding to the total density of fermions at $r=0$ when $g=0$, i.e., $n_{\uparrow}(0)+n_{\downarrow}(0)=k_{F}^{2} /(2 \pi)$. We also relate $\varepsilon_{c}$ and $\varepsilon_{F}$ to the occupation of harmonic oscillator levels as $\varepsilon_{c}=\omega\left(N_{c}+1\right)$ and $\varepsilon_{F}=\omega\left(N_{F}+1\right)$, respectively, where $N_{c} \gg N_{F}$. This leads to a total of $N=\left(N_{F}+1\right)\left(N_{F}+2\right)$ fermions, and therefore, $\varepsilon_{F} \approx \omega \sqrt{N}$ when $N_{F} \gg 1$. In addition, in order to be consistent with the energy cutoff, we choose $n_{\max }=\left(N_{c}-|m|\right) / 2$ as the maximum radial quantum number for a given $m$ and $m_{\max }=N_{c}$ as the maximum angular quantum number. In particular, here we choose $N_{F}=25$ and $\varepsilon_{c}=7 \varepsilon_{F}$, which correspond to a total of $N=702$ fermions and $N_{c}=181$. We checked that these values are sufficiently high for the parameter regime of our interest since our results agree well (within a few percent) with those obtained within the local-density approximation.

Population-balanced Fermi gases. In Fig. 1, we set $P=0$ and $\left|\varepsilon_{b}\right|=0.2 \varepsilon_{F}$, and show $\Delta(r)$ and $n_{\sigma}(r)$ for a number
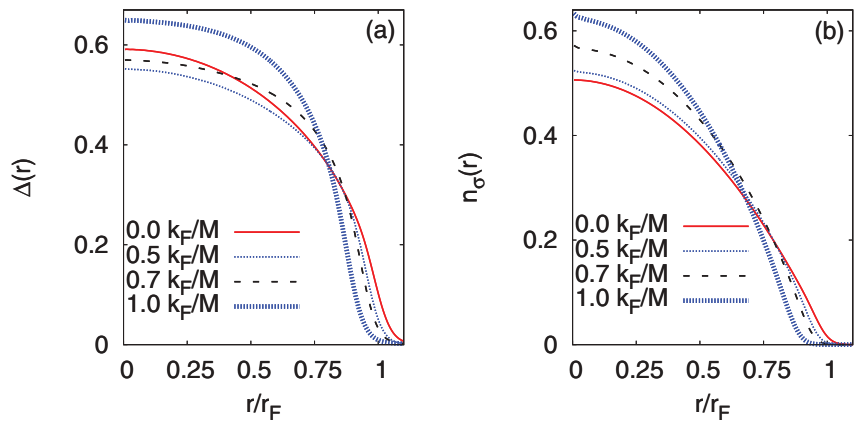

FIG. 1. (Color online) Balanced ( $P=0$ ) Fermi gas, where $\Delta(r)$ is in units of $\varepsilon_{F}$ and $n_{\sigma}(r)$ is in units of $k_{F}^{2} /(2 \pi)$.

of $\alpha$ values. First of all, since SOC increases the lowenergy density of states, which is similar to what happens in thermodynamic systems $[9,10]$, increasing $\alpha$ monotonically increases $n_{\sigma}(r)$ near $r=0$, and as a result, the Fermi gas shrinks. For instance, when $\alpha$ is increased from 0 to $k_{F} / M$, the central $n_{\sigma}(r)$ increases by $25 \%$, going from $k_{F}^{2} /(4 \pi)$ to approximately $5 k_{F}^{2} /(16 \pi)$. However, the corresponding $\Delta(r)$ has a nonmonotonic dependence on $\alpha$. We find that the central $\Delta(r)$ decreases slightly until a critical value of $\alpha \approx 0.5 k_{F} / M$ is reached, beyond which $\Delta(r)$ increases with increasing $\alpha$. The increase in $\Delta(r)$ is again mainly a consequence of increased density of states.

As we discuss below, the presence of a Rashba SOC spontaneously induces countercirculating mass currents. This is clearly seen in Fig. 2(a), where the $\uparrow$ and $\downarrow$ fermions are rotating around the center of the trap in opposite directions but with equal speed due to the time-reversal symmetry of the parent Hamiltonian. We note that the directions of circulating currents are determined by the chirality of the SOC, and the $\uparrow$ and $\downarrow$ currents would reverse directions if $K_{\uparrow \downarrow}(\mathbf{r})=\alpha\left(p_{y}-i p_{x}\right)$ is used. We see that $J_{\downarrow}(r)=-J_{\uparrow}(r)$ has a nonmonotonic dependence on $r$ : it gradually increases from zero as a function of $r$, making a peak at an intermediate distance near the edge of the system, beyond which it rapidly decreases to zero. The peak value of $J_{\sigma}(r)$ increases with increasing $\alpha$ since a nonzero $\alpha$ is what causes countercurrents to circulate to begin with. In addition, since increasing $\alpha$ shrinks the Fermi gas, the radial location of the peak moves inwards towards the trap center. Associated with this particle flow, we also find that the total angular momentum per particle
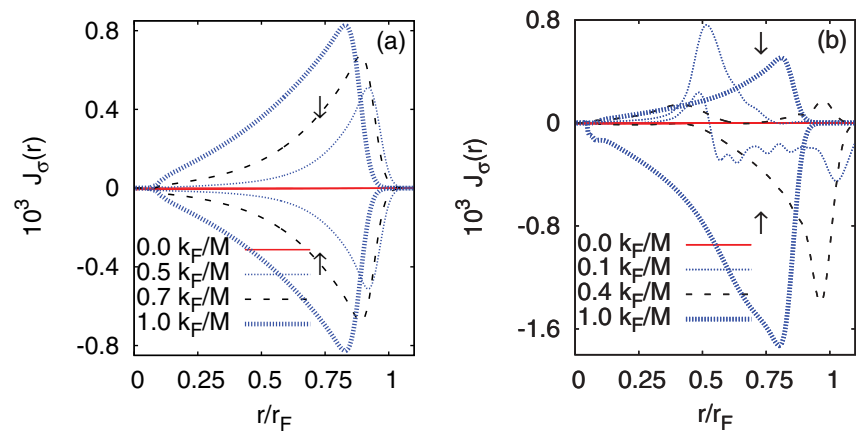

FIG. 2. (Color online) $J_{\sigma}(r)$ is shown in units of $k_{F}^{3} / M$ for the data of Figs. 1 and 3. 

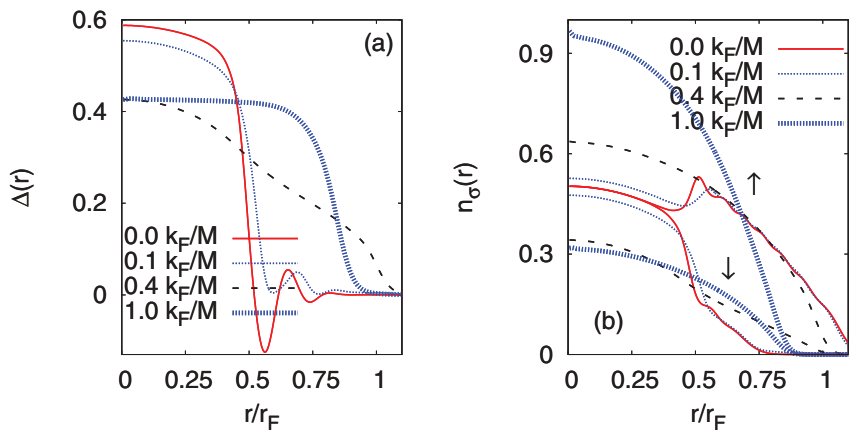

FIG. 3. (Color online) Imbalanced $(P=0.5)$ Fermi gas, where $\Delta(r)$ is in units of $\varepsilon_{F}$ and $n_{\sigma}(r)$ is in units of $k_{F}^{2} /(2 \pi)$.

$L_{\downarrow} / N_{\downarrow}=-L_{\uparrow} / N_{\uparrow}$ monotonically increases from zero and is bounded by 0.5 when $\alpha \gg k_{F} / M$ [27]. The origin of spontaneously induced countercirculating mass currents can be understood via a direct correspondence with the $p_{x}+$ $i p_{y}$ superfluids and superconductors [26]. In these $p$-wave systems, the mass current is associated with the chirality of Cooper pairs [28], and this is easily seen by noting that the chiral $p$-wave order parameter $\Delta_{\mathbf{k}} \propto(\hat{x} \pm i \hat{y}) \cdot \mathbf{k}$, where $\mathbf{k}$ is the relative momentum of a Cooper pair, is an eigenfunction of the orbital angular momentum with eigenvalue $\pm \hbar$. This explains our findings since it can be shown that the order parameter of Fermi gases with Rashba SOC and $s$-wave contact interactions has chiral $p$-wave symmetry [9]. However, unlike the chiral $p$-wave systems which break time-reversal symmetry and belong to the topological class of integer quantum Hall systems, spin-orbit-coupled Fermi gases preserve time-reversal symmetry just like quantum spin Hall systems, and therefore, they exhibit spontaneously induced countercirculating $\uparrow$ and $\downarrow$ mass currents.

Population-imbalanced Fermi gases. In Fig. 3, we set $P=0.5$ and $\left|\varepsilon_{b}\right|=0.2 \varepsilon_{F}$ and show $\Delta(r)$ and $n_{\sigma}(r)$ for a number of $\alpha$ values. When $\alpha=0$, we see that $n_{\uparrow}(r)=n_{\downarrow}(r)$ for $r \lesssim 0.25 r_{F}, n_{\uparrow}(r)>n_{\downarrow}(r) \neq 0$ for $0.25 r_{F} \lesssim r \lesssim 0.8 r_{F}$, and $n_{\uparrow}(r)>n_{\downarrow}(r)=0$ for $r \gtrsim 0.8 r_{F}$. Therefore, the central region corresponds to an unpolarized superfluid, and the excess spin-polarized $\uparrow$ fermions are expelled towards the edge of the system; i.e., paired $\uparrow$ and $\downarrow$ fermions and unpaired normal $\uparrow$ fermions are phase separated, with a coexistence region (i.e., a polarized superfluid) in between.

For small $\alpha \neq 0$, we see that the polarized-superfluid region rapidly expands towards the central region, and the system mostly consists of a polarized superfluid near $r=0$ which is phase separated from spin-polarized normal $\uparrow$ fermions residing near the edge. For larger $\alpha$ values, the spin-polarized $\uparrow$ gas gives way to the polarized superfluid, and the entire system eventually becomes a polarized superfluid beyond a critical $\alpha$. This happens around $\alpha \gtrsim 0.5 k_{F} / M$ when $P=0.5$ and $\left|\varepsilon_{b}\right|=0.2 \varepsilon_{F}$. We note in passing that these findings are consistent with the recent works on thermodynamic phase diagrams [14-19], where the phase-separated state was shown to become gradually unstable against the polarized superfluid phase as $\alpha$ increases from zero.

In addition, these recent works on thermodynamic systems showed that, unlike the $\alpha=0$ limit where the unpolarized superfluid phase is gapped and polarized superfluid phase is gapless, $\alpha \neq 0$ allows the possibility of having a gapped polarized superfluid phase up to a critical polarization, depending on the particular value of $\alpha$ [14-19]. Therefore, when $\alpha \neq 0$, in contrast to the topologically trivial unpolarized and low-polarized superfluid phases, the polarized superfluid phase with sufficiently high polarization becomes topologically nontrivial and has gapless excitations. Note that in a trapped system the topologically nontrivial locally high-polarized superfluid phase is sandwiched between topologically trivial phases (locally unpolarized or low-polarized superfluid and spin-polarized normal) for small $\alpha$.

The corresponding $\Delta(r)$ are shown in Fig. 3 (a). When $\alpha=0$, we see that $\Delta(r)$ oscillates with multiple sign changes, which is reminiscent of Fulde-Ferrel-Larkin-Ovchinnikov (FFLO) type spatially modulated superfluid phases. Similar to the $P=0$ case, for small $\alpha \neq 0$, the central $\Delta(r)$ decreases slightly until a critical value of $\alpha \approx 0.7 k_{F} / M$ is reached, beyond which $\Delta(r)$ increases with increasing $\alpha$. More importantly, the spatial modulations of $\Delta(r)$ rapidly disappear with increasing $\alpha$, and $\Delta(r)$ first becomes finite and then gradually increases near the edge of the system. This again indicates that the polarized superfluid phase expands towards the edge of the system as $\alpha$ gets larger. For larger $\alpha$ values, $\Delta(r)$ gradually increases everywhere, and it eventually becomes nearly flat for a substantial region of the system, except for a small region around the edge. These findings suggest that FFLO-type modulated phases, which are known to play a minor role in the thermodynamic phase diagrams when $\alpha=0$, become negligible for sufficiently large $\alpha$. Therefore, our work provides supporting evidence that the recent thermodynamic phase diagrams [14-19], where FFLO-type phases were entirely neglected, are qualitatively accurate at least within the mean-field approximation.

Since population imbalance breaks the time-reversal symmetry when $P \neq 0$, the $\uparrow$ and $\downarrow$ fermions again rotate (mostly) in opposite directions with unequal speeds, as seen in Fig. 2(b). Similar to the $P=0$ case, we again see that $\left|J_{\uparrow}(r)\right| \geqslant J_{\downarrow}(r)$ has a nonmonotonic dependence on $r$, and their peak values increase with increasing $\alpha$. We also find that $\left|L_{\uparrow}\right| / N_{\uparrow}>L_{\downarrow} / N_{\downarrow}$ increases from zero nonmonotonically, and they are bounded by 0.5 when $\alpha \gg k_{F} / M$ [27].

Inner-and outer-interface modes. In Fig. 4 , we show $\varepsilon_{m n}$ as a function of $m$ for imbalanced Fermi gases. First of all, we note that the spectrum satisfies $\varepsilon_{m n}=-\varepsilon_{-m-1, n}$, which follows
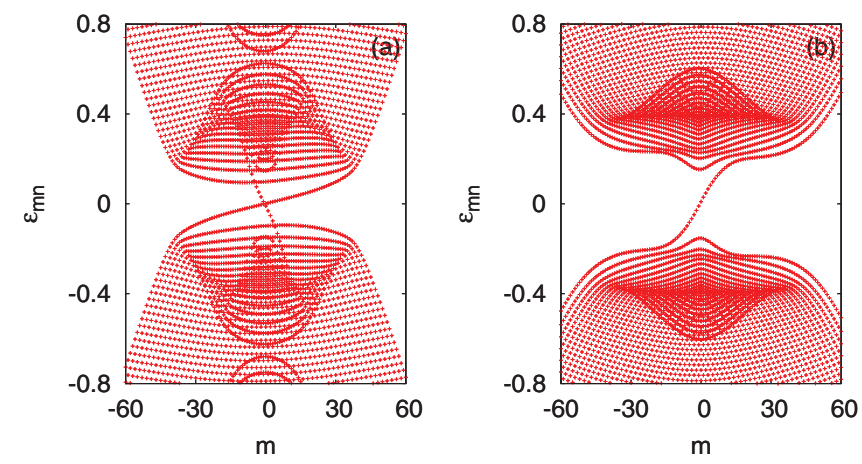

FIG. 4. (Color online) $\varepsilon_{m n}$ is shown in units of $\varepsilon_{F}$ for $\left|\varepsilon_{b}\right|=0.2 \varepsilon_{F}$ and $P=0.5$, where $\alpha$ is $0.4 k_{F} / M$ in (a) and $1.0 k_{F} / M$ in (b). 
from the particle-hole symmetry of the parent Hamiltonian. When $P=0$ and $\alpha=0$, it is well known that the quasiparticle and quasihole spectrum are separated with an energy gap around $m \approx 0$. When $P=0$ and $\alpha \neq 0$, it is expected that the spectrum splits in two in $m$ space, creating two identical energy gaps located at finite $m$ values. Their locations are approximately symmetric around $m=0$. For low $P \neq 0$ the spectrum is similar.

When $P \neq 0$ is sufficiently high and $\alpha$ is small, we show in Fig. 4(a) that the continuum of quasiparticle and quasihole spectrum is connected by two discrete branches, i.e., inner- and outer-interface modes [23]. This indicates that there must be two phase boundaries (interfaces) between a topologically nontrivial superfluid phase and a trivial one. In our case, while the inner mode occurs at the interface between the locally unpolarized or low-polarized superfluid phase existing near $r=0$ and the locally high-polarized superfluid phase existing at some intermediate region, the outer mode occurs at the interface between the locally high-polarized superfluid phase and locally spin-polarized normal phase existing near the edge of the system. However, the energy separation between the inner-interface modes becomes larger with increasing $\alpha$, which causes this branch to move completely into the continuum beyond a critical $\alpha$ value. Therefore, for large $\alpha$, the continuum of the quasiparticle and quasihole spectrum is connected by a single branch of outer-interface modes. This is clearly seen in
Fig. 4(b), and it is a direct consequence of the disappearance of the inner phase boundary, which approximately happens when $\alpha \gtrsim 0.5 k_{F} / M$, as discussed above.

Conclusions. To conclude, one of our main findings is that even a small SOC destabilizes FFLO-type superfluid phases against the polarized superfluid phase. This suggests that FFLO-type phases, which are known to play a minor role in the thermodynamic phase diagrams when $\alpha=0$, become negligible for sufficiently large $\alpha$. Therefore, we provided supporting evidence that the recent thermodynamic phase diagrams [14-19], where FFLO-type phases were entirely neglected, are qualitatively accurate at least within the mean-field approximation. We also found that the phaseseparated state rapidly becomes unstable against polarized superfluid phase as $\alpha$ increases from zero, which is in good agreement with recent works on thermodynamic phase diagrams. In addition, depending on the values of $P$ and $\alpha$, we showed for imbalanced Fermi gases that the continuum of the quasiparticle and quasihole excitation spectrum can be connected by zero, one, or two discrete branches of interface modes, the number of which is determined by the number of interfaces between a topologically trivial phase and a nontrivial phase.

Acknowledgments. This work is supported by Marie Curie Grant No. FP7-PEOPLE-IRG-2010-268239, TÜBITAK Grant No. 3501-110T839, and a TÜBA-GEBIP grant, and more details can be found in Ref. [27].
[1] M. Z. Hasan and C. L. Kane, Rev. Mod. Phys. 82, 3045 (2010).

[2] X.-L. Qi and S.-C. Zhang, Rev. Mod. Phys. 83, 1057 (2011).

[3] Y.-J. Lin, K. Jiménez-García, and I. B. Spielman, Nature (London) 471, 83 (2011).

[4] J.-Y. Zhang, S.-C. Ji, Z. Chen, L. Zhang, Z.-D. Du, B. Yan, G.-S. Pan, B. Zhao, Y.-J. Deng, H. Zhai, S. Chen, and J.-W. Pan, Phys. Rev. Lett. 109, 115301 (2012).

[5] P. Wang, Z.-Q. Yu, Z. Fu, J. Miao, L. Huang, S. Chai, H. Zhai, and J. Zhang, Phys. Rev. Lett. 109, 095301 (2012).

[6] L. W. Cheuk, A. T. Sommer, Z. Hadzibabic, T. Yefsah, W. S. Bakr, and M. W. Zwierlein, Phys. Rev. Lett. 109, 095302 (2012).

[7] E. Cappelluti, C. Grimaldi, and F. Marsiglio, Phys. Rev. Lett. 98, 167002 (2007).

[8] J. P. Vyasanakere, S. Zhang, and V. B. Shenoy, Phys. Rev. B 84, 014512 (2011).

[9] Z. Q. Yu and H. Zhai, Phys. Rev. Lett. 107, 195305 (2011); H. Zhai, Int. J. Mod. Phys. B 26, 1230001 (2012).

[10] H. Hu, L. Jiang, X.-J. Liu, and H. Pu, Phys. Rev. Lett. 107, 195304 (2011); L. Jiang, X.-J. Liu, H. Hu, and H. Pu, Phys. Rev. A 84, 063618 (2011).

[11] X. Yang and S. Wan, Phys. Rev. A 85, 023633 (2012).

[12] S. Takei, C.-H. Lin, B. M. Anderson, and V. Galitski, Phys. Rev. A 85, 023626 (2012).

[13] M. Gong, S. Tewari, and C. Zhang, Phys. Rev. Lett. 107, 195303 (2011); G. Chen, M. Gong, and C. Zhang, Phys. Rev. A 85, 013601 (2012).
[14] M. Iskin and A. L. Subaşı, Phys. Rev. Lett. 107, 050402 (2011); Phys. Rev. A 84, 043621 (2011).

[15] W. Yi and G.-C. Guo, Phys. Rev. A 84, 031608(R) (2011).

[16] L. Han and C. A. R. Sá de Melo, Phys. Rev. A 85, 011606(R) (2012); K. Seo, L. Han, and C. A. R. Sá de Melo, ibid. 85, 033601 (2012).

[17] K. Zhou and Z. Zhang, Phys. Rev. Lett. 108, 025301 (2012).

[18] R. Liao, Y. Yi-Xiang, and W.-M. Liu, Phys. Rev. Lett. 108, 080406 (2012).

[19] J. N. Zhang. Y. H. Chan, and L. M. Duan, arXiv:1110.2241.

[20] J. Zhou, W. Zhang, and W. Yi, Phys. Rev. A 84, 063603 (2011).

[21] S. K. Ghosh, J. P. Vyasanakere, and V. B. Shenoy, Phys. Rev. A 84, 053629 (2011).

[22] L. He and X. G. Huang, Phys. Rev. Lett. 108, 145302 (2012).

[23] X.-J. Liu, L. Jiang, H. Pu, and H. Hu, Phys. Rev. A 85, 021603(R) (2012)

[24] M. Feld, B. Frohlich, E. Vogt, M. Koschorreck, and M. Köhl, Nature (London) 480, 75 (2011); E. Vogt, M. Feld, B. Frohlich, D. Pertot, M. Koschorreck, and M. Köhl, Phys. Rev. Lett. 108, 070404 (2012).

[25] A. T. Sommer, L. W. Cheuk, M. J. H. Ku, W. S. Bakr, and M. W. Zwierlein, Phys. Rev. Lett. 108, 045302 (2012).

[26] E. Doko, A. L. Subaşı, and M. Iskin, Phys. Rev. A 85, 053634 (2012).

[27] M. Iskin, arXiv:1206.1240.

[28] T. Mizushima, M. Ichioka, and K. Machida, Phys. Rev. Lett. 101, 150409 (2008). 